\title{
Cutaneous epitheliotropic T-cell lymphoma with Sézary syndrome in a dog
}

\author{
Song in Lee ${ }^{1}$, Han Joon Lee ${ }^{1}$, Kyoungwon Seo ${ }^{2}$, Kunho Song ${ }^{1 *}$ \\ ${ }^{1}$ College of Veterinary Medicine, Chungnam National University, Daejeon 14134, Korea \\ ${ }^{2}$ College of Veterinary Medicine, Seoul National University, Seoul 08826, Korea
}

Received December 8, 2021

Revised December 24, 2021

Accepted December 24, 2021

Corresponding author:

Kunho Song

E-mail: songkh@cnu.ac.kr

https://orcid.org/0000-0001-8478-2035

\begin{abstract}
A 12-year-old, spayed female, Toy-poodle dog presented in progressive, pruritic carpal mass and mandibular papule. The skin lesions and enlarged superficial lymph nodes were surgically removed. According to histopathology and immunohistochemistry, the patient diagnosed as cutaneous epitheliotropic T-cell lymphoma with lymph node metastasis. Chemotherapy with L-asparaginase, lomustine and prednisolone was initiated. The patient deteriorated despite treatment and was admitted to the hospital. Blood tests revealed significant leukocytosis. Circulating large lymphocytes with convoluted and cerebriform nuclei seen in peripheral blood led to presumption of Sézary cells. Thus, considering skin, lymph nodes and blood involvement, Sézary syndrome was diagnosed.
\end{abstract}

Key Words: Toy-poodle dog, Cutaneous T-cell lymphoma, Sézary syndrome, Sézary cells

\section{INTRODUCTION}

Cutaneous epitheliotropic T-cell lymphoma (CETL) in dogs is a rare neoplasm characterized by infiltration of neoplastic $\mathrm{T}$ lymphocytes with a tropism specific to the epidermis and adnexal structures (Fontaine et al, 2009). Based on the clinical and histopathologic features, canine CETL can be divided into three subtypes using criteria described in humans. The three subtypes are mycosis fungoides (MF), pagetoid reticulosis (PR) and Sézary syndrome (Look, 2019).

Sézary syndrome is an end-stage leukemic form of cutaneous epitheliotropic T-cell lymphoma (CETL). In this syndrome, neoplastic lymphocytes called Sézary cells are found in peripheral blood in addition to erythematous skin lesions and peripheral lymphadenopathy (Vail, 2019). These cells can be identified on the basis of the characteristic convoluted, cerebriform nuclei, condensed nuclear chromatin, and the invisible nucleoli. The cells are medium-to-large sized with abundant amounts of nongranular cytoplasm (Naeim et al, 2008).
Sézary syndrome is rare in dogs. To the best of our knowledge, there are only a few reports in veterinary medicine, and none documented in South Korea. This syndrome has a poor prognosis as compared to other forms of CETL in humans (Willemze et al, 2005) and several veterinary cases (Williams et al, 2006; Rütgen et al, 2016). A detailed and careful examination of peripheral blood for the presence of Sézary cells is important for the assessment of prognosis.

\section{CASE}

A 12-year-old, spayed female, Toy-poodle dog $(2.2 \mathrm{~kg})$ had a left carpal mass $(2.7 \mathrm{~cm} \times 2 \mathrm{~cm})$ with a mandibular papule $(1 \mathrm{~cm} \times 0.7 \mathrm{~cm})$. Both were progressive, the left carpal mass was seen two months before admission to the local animal clinic (LAC), and the mandibular papule was noted two days before admission the LAC. The lesions were suspected to be soft tissue sarcoma based on the results of cytology at LAC. The dog was put on treatment with prednisolone, cephalexin, and famotidine for 
1 week. Despite the therapy, the skin lesions worsened, and the patient was referred to the Veterinary Medical Teaching Hospital of Chungnam National University.

On physical examination, the right mandibular lymph node was noted to be enlarged compared to the contralateral side. Moreover, the bilateral popliteal LNs were also enlarged. The left carpal mass was painful, pruritic and ulcerative, and the mandibular papule was nonpainful and pruritic (Fig. 1). The initial diagnostic evaluation included blood analysis, radiography, ultrasound and urinalysis. There were no remarkable abnormalities in the blood profile or peripheral blood film.

Diagnostic imaging (X-ray, CT) revealed enlargement of the left sublingual LN, left superficial cervical LN, bilateral prescapular LNs, and bilateral mandibular LNs.

Seven days after the patient was referred to our hos- pital, the mass, papule, and enlarged LNs were surgical excised. Surgically removed left carpal mass, mandibular papule, bilateral prescapular LNs, and right mandibular LN were submitted for histopathology and immunohistochemistry.

Histopathology confirmed that the mandibular papule and left carpal mass were cutaneous lymphoma (Fig. 2). The haired skin section contained infiltrative round cell neoplasms within the pre-existing dermal stroma. The neoplastic cells were noted to be infiltrating through the basal layer of epidermis as well as effacing the hair follicles, sebaceous glands and apocrine glands. LNs were replaced by neoplastic lymphocytes (Fig. 2).

According to immunohistochemistry, neoplastic cells in skin and lymph node were strongly positive to CD3 (a common marker of all $\mathrm{T}$ lymphocyte) (Fig. 3). Com-
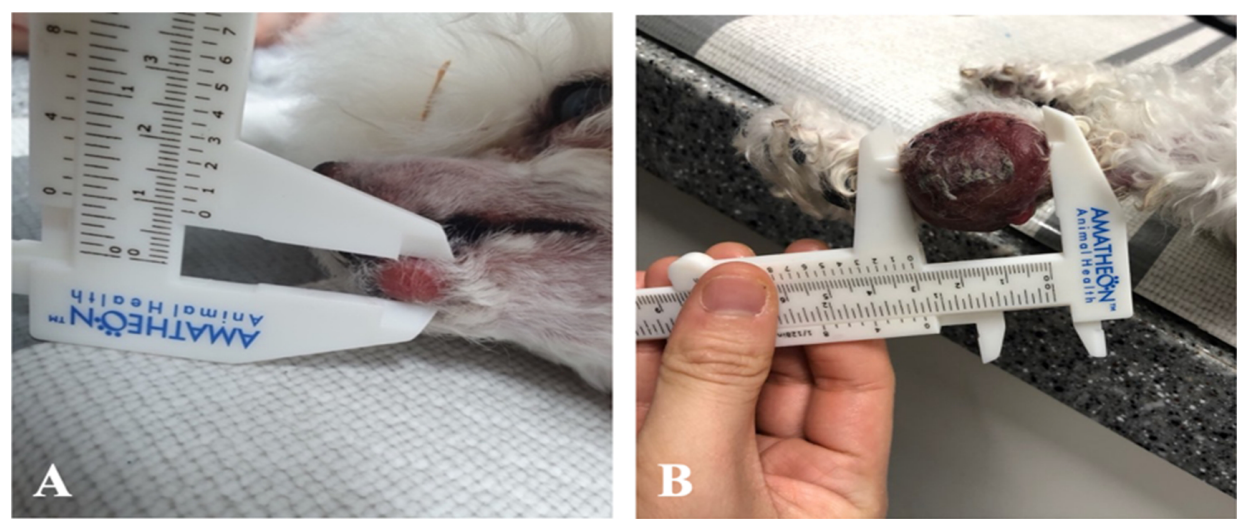

Fig. 1. In physical examination, mandibular papule (A) measuring $1 \mathrm{~cm} \times$ $0.7 \mathrm{~cm}$ and ulcerative carpal mass (B) measuring $2.7 \mathrm{~cm} \times 2 \mathrm{~cm}$ were noted.
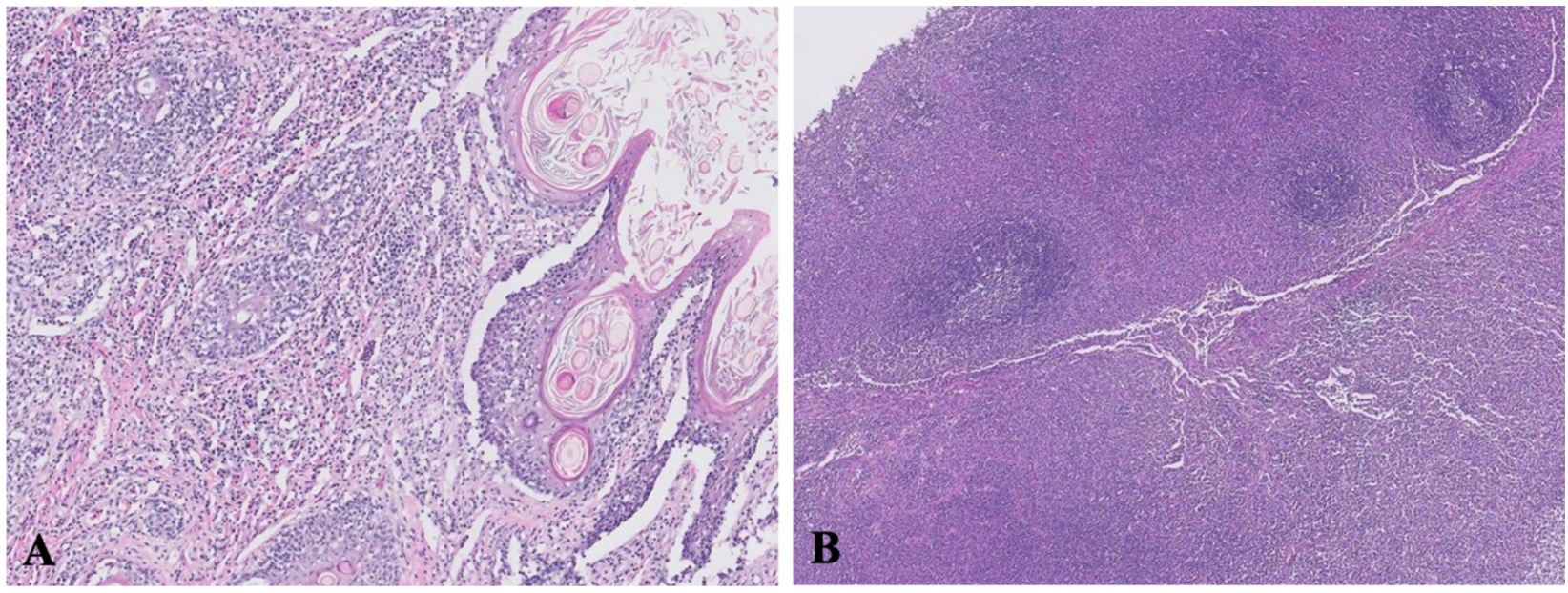

Fig. 2. Histopathologic result of left carpal mass (A) and right prescapular lymph node (B). Neoplastic cells are infiltrating through the basal layer of epidermis as well as effacing the hair follicles (A). Lymph node was partially replaced by neoplastic lymphocyte (B). 

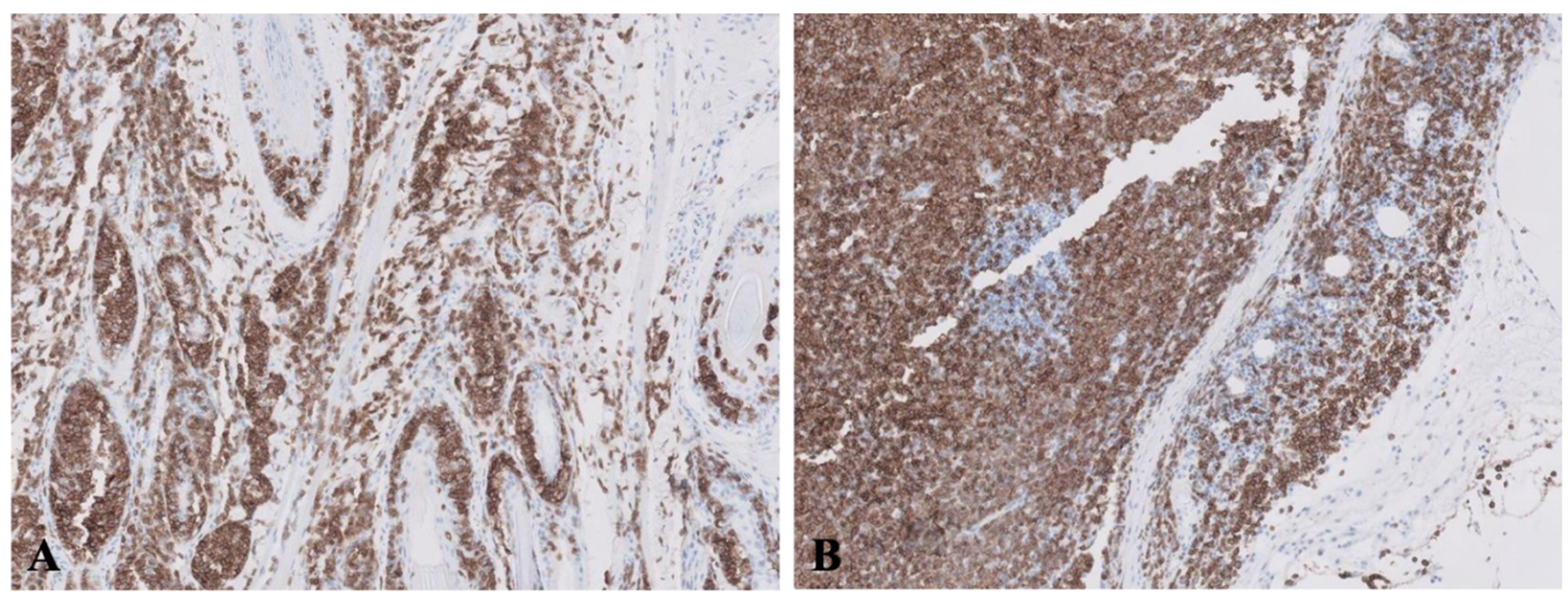

Fig. 3. Immunohistochemistry result (H\&E stained, CD3 antibody) of left carpal mass (A) and lymph node (B). Neoplastic cells are diffusely and strongly positive in the sections of haired skin (A) and lymph node (B).
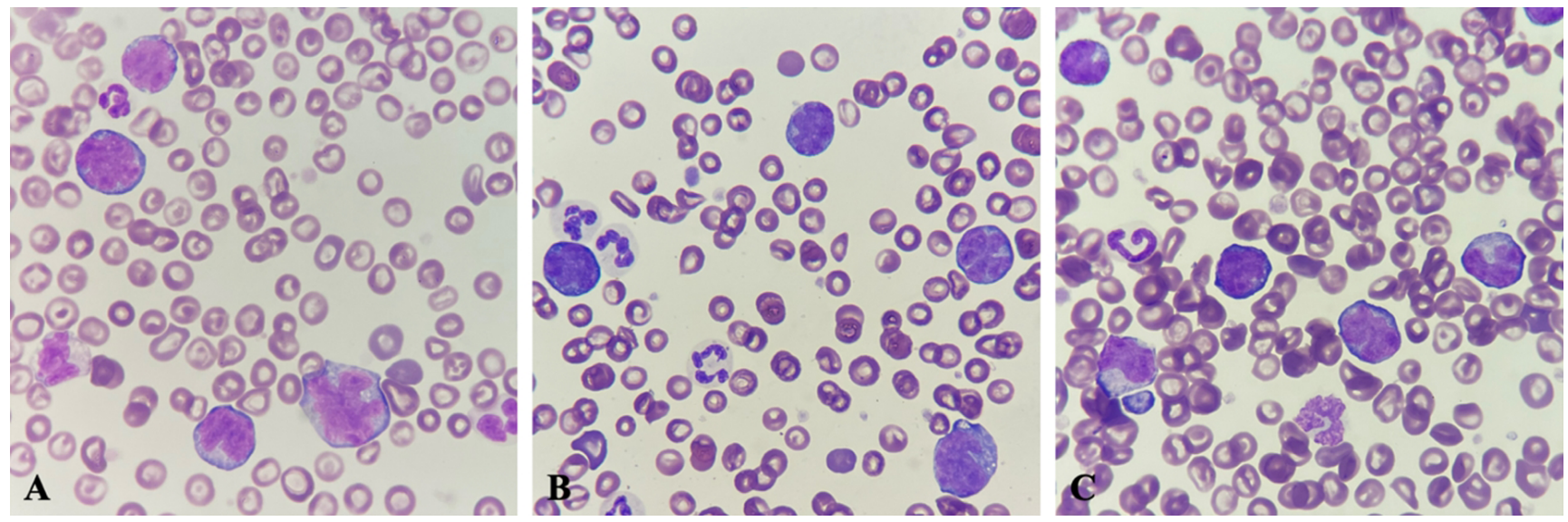

Fig. 4. Peripheral blood film on Day 40 (Wright's strain, $\times 1000)$. Large lymphocytes $(15 \sim 20 \mu \mathrm{m}$ in diameter, 3 times larger than RBC) with convoluted, cerebriform nucleus and basophilic cytoplasm are found.

bined with previously described in histopathology and immunohistochemistry stain results, the patient was diagnosed as cutaneous epitheliotropic T-cell lymphoma (CETL) that has spread to lymph nodes.

Based on the results of histopathology, 18 days after the patient was referred to our hospital, the first chemotherapy with L-asparaginase (Leunase inj., Kyowa Kirin S. Korea, 400 IU/kg, IV), prednisolone (Solondo tab, Korea Pharm, $2 \mathrm{mg} / \mathrm{kg}$ tapered to $1 \mathrm{mg} / \mathrm{kg}$, PO, SID) and lomustine $\left(70 \mathrm{mg} / \mathrm{m}^{2}\right.$, PO, ) was initiated. After chemotherapy, the patient showed neutropenia and hematochezia as side effects. However, the response to the therapy was assessed as progressive disease.

Forty days after referral to our hospital, the patient's con- dition deteriorated and she was re-hospitalized. Significant leukocytosis $\left(82.12 \times 10^{9} / \mathrm{L}\right.$, reference range: $5.2 \sim$ 13.9) and mild anemia (HCT, $28.1 \%$, reference range: 37.1 57) were observed. Except for mild azotemia (creatinine $2.1 \mathrm{mg} / \mathrm{dL}$ reference range: $0.3 \sim 1.3$, BUN $41.8 \mathrm{mg} / \mathrm{dL}$ reference range: $8 \sim 26$ ), other tests showed no abnormalities.

A peripheral blood smear revealed large lymphocytes $(15 \sim 20 \mu \mathrm{m})$ that were three times larger than red blood cells in the vicinity, and these cells had convoluted, folded, and cerebriform nuclei. The nucleus-to-cytoplasm ratio was high, and the cytoplasm of the overall cells was basophilic (Fig. 4). Based on the characteristics of the cells and the patient's underlying disease, we 
confirmed that they are Sézary cells.

In manual differential count, segmented neutrophils accounted for $49.5 \%$ of the total leukocytes, band neutrophils, lymphocytes and monocytes accounted for $2.5 \%, 22.5 \%$ and $6 \%$ of the total leukocytes. The remaining $19.5 \%$ of leukocytes were counted as Sézary cells. The patient died two days after examination of the peripheral blood. Necropsy was not performed because the owner was opposed to it.

\section{DISCUSSION}

CETL is highly variable, and the disease can mimic many inflammatory dermatoses. Although the etiology of CETL remains unclear, the most challenging aspect of diagnosis is the identification of the disease at an early stage (Fontaine et al, 2009). Of the 72 cases reported in dogs, 34 were female and 38 were male. The mean age at the time of diagnosis was 8.6 years (Thrall et al, 1984).

As per the available literature, reports show that English cocker spaniels and boxers may be predisposed to this disease (Thrall et al, 1984; Fontaine et al, 2009). Sézary syndrome is a generalized epitheliotropic T-cell lymphoma accompanied by erythroderma. To date, only a few reports describe Sézary syndrome in dogs, and the clinical presentation and morphologic features of the disease in dogs resemble their human counterparts (Rütgen et al, 2016). Sézary syndrome is a rare subtype of CETL, characterized by skin involvement with evidence of circulating large (15 20 $\mu \mathrm{m}$ in diameter) malignant $\mathrm{T}$ cells with folded, grooved nuclei in peripheral blood (Vail, 2019).

In this case, the patient had CETL and had systemic lymphadenopathy confirmed as metastatic lymph nodes based on histopathology and immunohistochemistry. In addition, as characteristic large, convoluted, cerebriform cells were found in peripheral blood, we diagnosed this patient with Sézary syndrome according to the definition described above.

Sézary syndrome is a progressive disease form of CETL (Rook, 2019). When determining the degree of exacerbation of cutaneous T-cell lymphoma in human medicine, Tumor-Node-Metastasis-Blood staging is used, in which the blood stage is divided by the number of Sézary cells in the peripheral blood (Willemze et al, 2005).

In this case, the initial blood tests or blood smears of this patient showed no suspicion of Sézary syndrome. The Sézary syndrome probably developed as the disease progressed, and was diagnosed through cytologic features of the peripheral blood smear.

Treatment for diffuse CETL is best managed with systemic therapy. The most widely used chemotherapy protocols for CETL include lomustine along with prednisolone, pegylated l-asparaginase, and oral retinoic acid analogs (Vail, 2019). The reported response rates range from $40 \%$ to $80 \%$ (Willemze et al, 2005).

In this case, the patient was evaluated as progressive disease because there was no response to chemotherapy, even though the patient received chemotherapy immediately after removal of a large skin lesion by surgery.

The prognosis for Sézary syndrome is yet to be accurately evaluated in veterinary medicine. However, in human medicine, a series of studies have demonstrated that a high blood tumor burden with more absolute Sézary cells is associated with a worse prognosis (Willemze et al, 2005). In addition, similar Sézary syndrome case reports in veterinary medicine have documented a short survival period (37 days after diagnosis in a 10year Scottish terrier, 2 days after diagnosis in a 15year cat) (Thrall et al, 1984; Fontaine et al, 2009). In this case, the patient died 2 days after being diagnosed with Sézary syndrome. This suggests that Sézary syndrome has a worse prognosis in veterinary medicine which needs to be studied further.

In conclusion, this is the first case of Sézary syndrome in a dog from South Korea. Because Sézary syndrome is a progressive form of CETL and associated with a worse prognosis, careful examination of the presence of Sézary cells in peripheral blood is important in diagnosing and subsequent treatment of CETL. 


\section{CONFLICT OF INTEREST}

No potential conflict of interest relevant to this article was reported.

\section{ORCID}

Song in Lee, https://orcid.org/0000-0001-6937-1422

Han Joon Lee, https://orcid.org/0000-0001-9340-4095

Kyoungwon Seo, https://orcid.org/0000-0002-1561-3278

Kunho Song, https://orcid.org/0000-0001-8478-2035

\section{REFERENCES}

Fontaine J, Bovens C, Bettenay S, Mueller RS. 2009. Canine cutaneous epitheliotropic T-cell lymphoma: a review. Vet Comp Oncol 7: 1-14.

Naeim F, Pao P, Song S, Phan R. 2008. Atlas of Hematopathology. 2nd ed. Pp. 693-700, Academic Press, Salt Lake.

Rook KA. 2019. Canine and feline cutaneous epitheliotropic lymphoma and cutaneous lymphocytosis: Vet Clin North Am Small Anim Pract 49: 67-81.

Rütgen BC, Flickinger I, Wolfesberger B, Litschauer B,
Fuchs-Baumgartinger A, Hammer SE, Saalmüller A, Schwendenwein I. 2016. Cutaneous T-cell lymphoma-Sézary Syndrome in a Boxer. Vet Clin Pathol 45: 172-178.

Thrall MA, Macy DW, Snyder SP, Hall RL. 1984. Cutaneous lymphosarcoma and leukemia in a dog resembling Sezary syndrome in man. Vet Pathol 21: 182186.

Vail DM. 2019. Canine lymphoma and lymphocytic leukemia. Pp. 688-711. In: Vail DM, Thamm DH, Liptak JM (ed.). Withrow and MacEwen's small animal clinical oncology, 6th ed. Elsevier, St. Louis.

Willemze R, Jaffe ES, Burg G, Cerroni L, Berti E, Swerdlow SH, Ralfkiaer E, Chimenti S, Diaz-Perez JL, Duncan LM, Grange F, Harris NL, Kempf W, Kerl H, Kurrer M, Knobler R, Pimpinelli N, Sander C, Santucci M, Sterry W, Vermeer MH, Wechsler J, Whittaker S, Meijer CJ. 2005. WHO-EORTC classification for cutaneous lymphomas. Blood 105: 37683785.

Williams LE, Rassnick KM, Power HT, Lana SE, Morrison-Collister KE, Hansen K, Johonson JL. 2006. CCNU in the treatment of canine epitheliotropic lymphoma, J Vet Intern Med 20: 136-143. 